\title{
SELBERG ZETA-FUNCTION ASSOCIATED TO COMPACT RIEMANN SURFACE IS PRIME
}

\author{
RAMŪNAS GARUNKŠTIS
}

\begin{abstract}
Let $Z(s)$ be the Selberg zeta-function associated to a compact Riemann surface. We consider decompositions $Z(s)=f(h(s))$, where $f$ and $h$ are meromorphic functions, and show that such decompositions can only be trivial.
\end{abstract}

\section{INTRODUCTION}

We continue the investigation of decompositions of the Selberg zeta-function which was started in Garunkštis and Steuding [6]. First we reproduce required definitions. Let $s=\sigma+i t$ be a complex variable and $X$ a compact Riemann surface of genus $g \geq 2$ with constant negative curvature -1 . The surface $X$ can be written as a quotient $\Gamma \backslash H$, where $\Gamma \subset \operatorname{PSL}(2, \mathbb{R})$ is a strictly hyperbolic Fuchsian group and $H$ is the upper half-plane of $\mathbb{C}$. Then the Selberg zeta-function associated with $X=\Gamma \backslash H$ is defined by (see Hejhal [8, $\S 2.4$, Definition 4.1])

$$
Z(s)=\prod_{\left\{P_{0}\right\}} \prod_{k=0}^{\infty}\left(1-N\left(P_{0}\right)^{-s-k}\right) .
$$

Here $\left\{P_{0}\right\}$ is the conjugacy class of a primitive hyperbolic element $P_{0}$ of $\Gamma$ and $N\left(P_{0}\right)=\alpha^{2}$ if the eigenvalues of $P_{0}$ are $\alpha$ and $\alpha^{-1}$ with $|\alpha|>1$. Equation (1.1) defines the Selberg zeta-function in the half-plane $\sigma>1$. The function $Z(s)$ can be extended to an entire function (see [8, § 2.4, Theorem 4.25]).

Definition 1.1 (Gross [7], Chuang and Yang [1, Section 3.2], [6]). Let $F$ be a meromorphic function. Then an expression

$$
F(z)=f(h(z)),
$$

where $f$ is meromorphic and $h$ is entire ( $h$ may be meromorphic when $f$ is a rational function), is called a decomposition of $F$, with $f$ and $h$ as its left and right components, respectively. $F$ is said to be prime in the sense of a decomposition

2020 Mathematics Subject Classification. Primary: 11M36; Secondary: 30D99.

Key words and phrases. Selberg zeta-function, decompositions of meromorphic functions, prime function, $a$-points.

This research is funded by the European Social Fund (project no. 09.3.3-LMT-K-712-01-0037) under grant agreement with the Research Council of Lithuania (LMTLT). 
if for every representation of $F$ of the form 1.2 we have that either $f$ or $h$ is linear. If every representation of $F$ of the form (1.2) implies that $f$ is rational or $h$ is a polynomial, we say that $F$ is pseudo-prime in the sense of a decomposition. Furthermore, $F$ is said to be left-prime (right-prime) if every factorization 1.2 . implies that $f$ is linear whenever $h$ is transcendental ( $h$ is linear whenever $f$ is transcendental).

Liao and Yang [10] showed that the Riemann zeta-function is prime. In [6] the following theorem is proved.

Theorem A. The Selberg zeta-function $Z$ associated with a compact Riemann surface of genus $g$ is pseudo-prime and right-prime. Moreover, if $Z(s)=f(h(s))$, where $f$ is rational and $h$ is meromorphic, then $f$ is a polynomial of degree $k$, where $k$ divides $2 g-2$, and $h$ is an entire function.

Here we complete Theorem A.

Theorem 1.2. The Selberg zeta-function $Z$ associated with a compact Riemann surface of genus $g \geq 2$ is prime.

Theorem 1.2 follows from Theorem A, the property that $Z(s)$ has a simple zero at $s=1([8, \S 2.4$, Theorem 4.11]), and the following lemma.

Lemma 1.3. If there exist a polynomial $P$ and an entire function $h$ such that $Z(s)=P(h(s))$ then the polynomial $P$ has only one root in the complex plane (counting without multiplicities).

The proof of Lemma 1.3 is based on the distribution of zeros of $Z(s)-a, a \in \mathbb{C}$, (such zeros are called $a$-points of $Z(s))$ and of zeros of $Z^{\prime}(s)$ in the left half-plane of $\mathbb{C}$. These zeros are described below.

The Selberg zeta-function $Z(s)$ has trivial zeros at integers $s=-n, n \geq 1$, of multiplicity $(2 g-2)(2 n+1)$; at $s=0$ with multiplicity $2 g-1$; and an already mentioned zero at $s=1$ with multiplicity 1 (see [8, $\S 2.4$, Theorem 4.11], also for nontrivial zeros).

For the trivial zeros of $Z^{\prime}(s)$, Theorem 1 from [5] together with the equality $\overline{Z(s)}=Z(\bar{s})$ give the following proposition.

\section{Proposition 1.4.}

(i) There is $\sigma_{0} \geq 1$ such that $Z^{\prime}(s) \neq 0$ in $\sigma \geq \sigma_{0}$;

(ii) the function $Z^{\prime}(s)$ has zeros at $s=n$ of multiplicity $(2 g-2)(1-2 n)-1$ for any $n \leq-1$, and at $s=0$ of multiplicity $2 g-2$.

Moreover, for any $0<\varepsilon<1 / 2$, there is a constant $n_{0}=n_{0}(\varepsilon) \leq-1$ such that

(iii) $Z^{\prime}(s)$ has a simple real zero in the disc $|s+1 / 2-n| \leq \varepsilon$ for any $n \leq n_{0}$;

(iv) $Z^{\prime}(s)$ has no other zeros in $\sigma \leq n_{0}$ except those mentioned in (ii) and (iii).

For more about the zeros of the derivative of the Selberg zeta-function see [4, 11, 12 .

For the $a$-points of $Z(s)$ we will prove the following two statements. 
Proposition 1.5. Let $b>0$ and $1 / 6<r<1 / 2$. Then there exists a negative number $N=N(Z, b, r)$ such that, for $a \in \mathbb{C}, 0<|a| \leq b$, the function $Z(s)-a$ has $(2 g-2)(1-2 n)$ simple zeros in $|s-n|<r$, where $n<N$ are integers. Furthermore, $Z(s)-a$ has no other zeros in $\sigma<N$.

On the other hand, Proposition 1.4 implies that, for sufficiently large negative $n$, a neighborhood of $n+1 / 2$ contains a double zero of $Z(s)-Z(n+1 / 2)$.

Using Proposition 1.5 and the particular kind of polynomials $P(z)=z^{k}+C$ we can easily demonstrate the main idea of the proof of Lemma 1.3 Indeed, let

$$
Z(s)=h(s)^{k}+C,
$$

where $C \neq 0$ and $h(s)$ is an entire function. Then all zeros of $Z(s)-C$ are at least of order $k$. By Proposition 1.5 we see that $k=1$ and Lemma 1.3 is true for this particular kind of polynomials. To consider the general case we will need the following consequence of Proposition 1.5 .

Corollary 1.6. Let $a:[0,1] \rightarrow \mathbb{C} \backslash 0$ be a continuous function. Then for any sufficiently large negative $n$ there are $(2 g-2)(2 n+1)$ continuous functions $s_{j}$ : $[0,1] \rightarrow \mathbb{C}$ such that, for each $j$, we have $Z\left(s_{j}(x)\right)=a(x),\left|s_{j}(x)-n\right|<1 / 3$, and $s_{j}(x) \neq s_{m}(x)$ if $j \neq m$ and $x \in[0,1]$.

In the last corollary, $1 / 3$ can be replaced by any number $r, 1 / 6<r<1 / 2$. Various properties of $a$-points of Selberg zeta-functions were considered in [2, 3].

The next section contains the proofs of Proposition 1.5. Corollary 1.6, and Lemma 1.3

\section{Proofs}

Proof of Proposition 1.5. We have (see [8, § 2.4, Theorem 4.12])

$$
Z(s)=f(s) Z(1-s),
$$

where

$$
f(s)=\exp \left(\operatorname{area}(X) \int_{0}^{s-1 / 2} v \tan (\pi v) d v\right) .
$$

It is known ([5, Lemma 6]) that, for $t \geq 0$ and $s$ not an integer,

$$
\begin{aligned}
& \int_{0}^{s-1 / 2} v \tan (\pi v) d v \\
& \quad=\frac{i(s-1 / 2)^{2}}{2}-\frac{s-1 / 2}{\pi} \log \left(1+e^{2 \pi i(s-1 / 2)}\right)+\frac{i}{2 \pi^{2}} \operatorname{Li}_{2}\left(-e^{2 \pi i(s-1 / 2)}\right)+\frac{i}{24},
\end{aligned}
$$

where the integration is along the straight line segment joining the origin to $s-1 / 2$ if $s$ is not on the real line; if $s$ is on the real line, and not an integer, we define the integral by the requirement of continuity as $s$ is approached from the upper half-plane; furthermore, the branch of the logarithm is chosen such that

$$
-\pi / 2 \leq \Im \log \left(1+e^{2 \pi i(s-1 / 2)}\right) \leq \pi / 2 .
$$


Then, for $\sigma \rightarrow-\infty$,

$$
\begin{aligned}
& |f(s)| \\
& \quad=\exp \left(\operatorname{area}(X)\left(-(\sigma-1 / 2) t-\frac{\sigma-1 / 2}{\pi} \log \left|1+e^{2 i \pi(s-1 / 2)}\right|+O(|t|+1)\right)\right)
\end{aligned}
$$

uniformly in $t \geq 0$. Let

$$
g(\sigma, t)=t+\frac{1}{\pi} \log \left|1+e^{2 i \pi(\sigma-1 / 2+i t)}\right| .
$$

We will observe that there is $\delta_{r}>0$ such that

$$
g(\sigma, t)>\delta_{r},
$$

where $s=\sigma+i t$ lies on the semicircle $|s-n|=r, t \geq 0, n \in \mathbb{Z}$, and $1 / 6<r<1 / 2$. Note that $g(x+n, t)=g(-x+n, t), x \in \mathbb{R}$. Thus it is enough to prove $(2.3)$ for the following quarter of the circle: $|s-n|=r, t \geq 0,0 \leq \sigma-n \leq r$, which we parametrize by $t=x, \sigma=\sqrt{r^{2}-x^{2}}+n, x \in[0, r]$. Consequently we consider the function

$$
q(x)=g\left(\sqrt{r^{2}-x^{2}}+n, x\right) .
$$

Straightforward calculations show that $q(0)>0$ and $q^{\prime}(x)>0$ for $0 \leq x \leq r$, $1 / 6<r \leq 1 / 2$. This establishes the inequality (2.3).

Hence, for any given real positive number $Y$ and $1 / 6<r<1 / 2$, there is a negative number $M=M(Y, r)$ such that

$$
|f(s)|=\exp (\operatorname{area}(X)(-(\sigma-1 / 2) g(\sigma, t)+O(|t|+1)))>Y,
$$

if $|s-n|=r, t \geq 0$, and $n<M$. The Dirichlet series expansion of $Z(s)$ yields

$$
|Z(s)|>1 / 2
$$

if $\sigma$ is sufficiently large. Note that

$$
\overline{Z(s)}=Z(\bar{s}) .
$$

Then Rouché's theorem gives that for sufficiently large negative $n$ the functions $Z(s)$ and $Z(s)-a$ have the same number of zeros in the disc $|s-n| \leq r$. In this disc $Z(s)$ has only one distinct zero at $s=n$ and clearly $Z(n) \neq a$. This, $1 / 6<r<1 / 2$, and Proposition 1.4 give that $Z(s)-a$ and $(Z(s)-a)^{\prime}=Z^{\prime}(s)$ have no common zeros in $\sigma<N$. Accordingly, all zeros of $Z(s)-a$ located in $|s-n| \leq r$ are simple.

It remains to show that for any sufficiently large negative $n$ the area $\{s:|s-n|>$ $r, n-1 / 2 \leq \sigma \leq n+1 / 2\}$ is free from zeros of $Z(s)-a$. This follows by the inequalities $\partial g(\sigma, t) / \partial t>0$ if $t>0, \sigma \in \mathbb{R}$ and $g(\sigma, 0)>0$ if $|\sigma-1 / 2-n|<1 / 3$, together with formulas $(2.1-2.5)$. Proposition 1.5 is proved. 
Lemma 2.1. If the polynomial $P(z)$ has at least two different roots, then there is a nonzero constant $c$ such that $P(z)-c$ has a multiple root.

Proof. Let $\operatorname{deg} P=k \geq 2$. Conversely to the statement of the lemma, suppose that the roots of $P(z)-c$ are simple for all $c \neq 0$. Then $(P(z)-c)^{\prime}=P^{\prime}(z)$ has no common roots with $P(z)-c$ for any $c \neq 0$. Therefore, for any root $z_{j}^{\prime}$, $j \in\{1, \ldots, k-1\}$, of $P^{\prime}(z)$, we have $P\left(z_{j}^{\prime}\right)=0$. This is possible only if $P(z)=$ $a\left(z-z_{1}^{\prime}\right)^{k}$ and $z_{j}^{\prime}=z_{1}^{\prime}$, for all $j \in\{2, \ldots, k-1\}$. The contradiction obtained proves the lemma.

Proof of Corollary 1.6. By Proposition 1.5. for any large negative $n$ and fixed $x \in$ $[0,1]$, there are exactly $(2 g-2)(n+1)$ simple zeros $s_{j}(x)$ of $Z(s)-a(x)$ in the disc $|s-n|<1 / 3$. Then the corollary follows by the implicit function theorem ([9, Theorem 2.4.1]) from which we see that $Z(s)$ is a one-to-one function in some neighborhood of each $s_{j}(x), j=1, \ldots,(2 g-2)(n+1), x \in[0,1]$.

Proof of Lemma 1.3. Note that $P$ cannot be a constant polynomial. To obtain a contradiction, assume that $Z(s)=P(h(s))$ and the polynomial $P, \operatorname{deg} P=k$, has at least two different roots. Then Lemma 2.1 implies the existence of $a_{1}$ such that $P^{\prime}\left(a_{1}\right)=0$ and $P\left(a_{1}\right) \neq 0$. Therefore we can write

$$
P(z)-P\left(a_{1}\right)=d\left(z-a_{1}\right)^{k_{1}} \ldots\left(z-a_{m}\right)^{k_{m}},
$$

where $k_{1} \geq 2$ and $k_{1}+\cdots+k_{m}=k$. In view of Proposition 1.5 there are infinitely many zeros of $Z(s)-P\left(a_{1}\right)$ each of which lies at a distance smaller than $1 / 3$ from some negative integer. Thus there are an infinite subset $S$ of these zeros and $a_{j}$ defined by 2.6 such that $h(\rho)-a_{j}=0$ for $\rho \in S$. If $k_{j} \geq 2$ then the zeros $\rho$ are multiple zeros of $Z(s)-P\left(a_{1}\right)$ and this contradicts Proposition 1.5 Hence $k_{j}=1$, $P^{\prime}\left(a_{j}\right) \neq 0$, and by 2.6 we see that $j \geq 2$. Therefore there is a continuous function $a:[0,1] \rightarrow \mathbb{C}$, such that $a(0)=a_{j}, a(1)=a_{1}$, and

$$
P^{\prime}(a(x)) \neq 0 \text { for } x \in[0,1) .
$$

By Corollary 1.6 there is a continuous function $\psi:[0,1] \rightarrow \mathbb{C}$ such that $\psi(0) \in S$,

$$
Z(\psi(x))=P(a(x)),
$$

and, for $x \in[0,1]$ and some large negative integer $n$,

$$
|\psi(x)-n|<1 / 3 .
$$

Note that $Z(\psi(x))=P(h(\psi(x)))$. To get the contradiction we will show that $h(\psi(1))=a_{1}$. By $(2.8)$

$$
P(h(\psi(x)))=P(a(x)) .
$$

In view of 2.7 we have that $P(z)$ is a one-to-one function in a sufficiently small neighborhood of any $a(x), x \in[0,1)$. Then $h(\psi(0))=a(0)$ leads to the equality $h(\psi(x))=a(x)$ for $x \in[0,1)$. Continuity gives $h(\psi(1))=a(1)=a_{1}$ and thus $z=\psi(1)$ is a multiple zero of $Z(z)-Z(\psi(1))$. This contradicts Proposition 1.5 and proves Lemma 1.3 


\section{REFERENCES}

[1] C.-T. Chuang and C.-C. Yang, Fix-points and factorization of meromorphic functions, World Scientific Publishing Teaneck, NJ, 1990. Translated from the Chinese. MR 1050548

[2] R. Garunkštis and R. Šimėnas, The $a$-values of the Selberg zeta-function, Lith. Math. J. 52 (2012), no. 2, 145-154. MR 2915767

[3] R. Garunkštis, J. Steuding and R. Šimènas, The $a$-points of the Selberg zeta-function are uniformly distributed modulo one, Illinois J. Math. 58 (2014), no. 1, 207-218. MR 3331847

[4] R. Garunkštis, Note on zeros of the derivative of the Selberg zeta-function, Arch. Math. (Basel) 91 (2008), no. 3, 238-246. MR 2439597. Corrigendum, Arch. Math. (Basel) 93 (2009), no. 2, 143-145.

[5] R. Garunkštis, Zero-free regions for derivatives of the Selberg zeta-function, Publ. Math. Debrecen 93 (2018), no. 3-4, 369-385. MR 3875342

[6] R. Garunkštis and J. Steuding, On primeness of the Selberg zeta-function, Hokkaido Math. J. 49 (2020), no. 3, 451-462. MR 4187117.

[7] F. Gross, On factorization of meromorphic functions, Trans. Amer. Math. Soc. 131 (1968), 215-222. MR 0220936

[8] D. A. Hejhal, The Selberg trace formula for $\operatorname{PSL}(2, R)$. Vol. I, Lecture Notes in Mathematics, Vol. 548, Springer-Verlag, Berlin, 1976. MR 0439755

[9] S. G. Krantz and H. R. Parks, The implicit function theorem, Modern Birkhäuser Classics, Birkhäuser/Springer, New York, 2013. MR 2977424

[10] L. Liao and C.-C. Yang, On some new properties of the gamma function and the Riemann zeta function, Math. Nachr. 257 (2003), 59-66. MR 1992811

[11] W. Luo, On zeros of the derivative of the Selberg zeta function, Amer. J. Math. 127 (2005), no. 5, 1141-1151. MR 2170140

[12] M. Minamide, The zero-free region of the derivative of Selberg zeta functions, Monatsh. Math. 160 (2010), no. 2, 187-193. MR 2644220

Ramūnas Garunkštis

Institute of Mathematics, Faculty of Mathematics and Informatics, Vilnius University,

Naugarduko 24, 03225 Vilnius, Lithuania

ramunas.garunkstis@mif .vu. It

$U R L:$ http://www.mif.vu.It/ garunkstis

Received: August 9, 2019

Accepted: April 21, 2020 